\title{
Split-course chemoradiotherapy with S-1, a novel oral fluorour- acil, and cisplatin for distant metastases of oesophageal cancer stage IVb
} Hiroaki Iwase ${ }^{1, *}$, Masaaki Shimada ${ }^{1}$, Noboru Hirashima ${ }^{1}$, Nobumitsu Ryuge ${ }^{1}$, Noboru Urata ${ }^{1}$, Momoko Goto ${ }^{1}$, Takashi Kondo ${ }^{1}$, Daiki
Tanaka', Satoshi Unita ${ }^{1}$ and Eriko Kato ${ }^{2}$

1 Departments of Gastroenterology, National Hospital Organization, Nagoya Medical Centre, Nagoya, Japan

2 Department of Radiology, National Hospital Organization, Nagoya Medical Centre, Nagoya, Japan

\begin{abstract}
Objectives: To evaluate the efficacy and safety of split-course chemoradiotherapy with S-1, a novel oral fluorouracil, together with cisplatin in patients with distant oesophageal cancer stage IVb metastasis. Methods: Forty-one patients with distant oesophageal cancer metastasis and performance status 0 or 1 received split-course chemoradiotherapy with S-1 and cisplatin. All 41 patients were reviewed retrospectively. Chemoradiotherapy comprised two courses of 30-Gy radiotherapy over three weeks plus daily oral S- 1 (70 $\left.\mathrm{mg} / \mathrm{m}^{2} / \mathrm{day}\right)$ for two weeks and a $24 \mathrm{~h}$ cisplatin infusion $\left(70 \mathrm{mg} / \mathrm{m}^{2}\right)$ on Day 8 , with a two week interval between the two courses. Results: The most frequent adverse events (AEs) were grade 3 and 4 neutropenia (29.2\%), thrombocytopenia (9.8\%), and anaemia (7.3\%). Non-haematological AEs were generally mild. AEs in the initial course of chemoradiotherapy remitted during the second interval week. Overall, the complete response rate was $22.0 \%$ and endoscopic complete response rate for primary lesion was $65.9 \%$. Thirty-one patients (75.6\%) became asymptomatic and regained normal swallowing function. The overall median survival time was 12 months. Conclusion: This retrospective investigation showed that split-course chemoradiotherapy with S-1 and cisplatin had an encouraging safety profile together with good efficacy. Potentially, this regimen may become a standard for distant metastasis of oesophageal cancer stage IVb.
\end{abstract}

Keywords: oesophageal cancer stage IVb; split-course chemoradiotherapy; S-1; cisplatin; swallowing function; prognosis

\section{Introduction}

Oesophageal cancer $(\mathrm{OC})$ is a highly malignant, but treatable disease. However, in spite of this understanding, at the time of diagnosis, more than $60 \%$ of patients with OC are incurable [1]. Multimodal therapy like chemoradiotherapy (CRT) or surgery followed by chemotherapy/CRT has been found to be effective in patients with locally advanced OC [2]. Unfortunately, at present there is no recommended treatment for patients with distant metastasis. When a patient's prognosis has not been judged adequately, he or she is considered to be a candidate for palliative therapy [3]. Patients' most stressful morbidity is dysphagia, or inability to swallow food, which severely affects the patients' quality of life and may lead to complications, including malnutrition and aspiration pneumonia. The quality of life of these patients depends to a large degree on their ability to swallow food, and normal swallowing function plays a vital role in the palliation of this disease. Several treatment strategies are applied for the palliation of dysphagia, including surgical bypass, chemotherapy, stent placement, laser ablation, and radiotherapy. Among these, radiotherapy has the advantages of being noninvasive and having a direct effect on the tumour, thereby improving the patient's swallowing ability [4].
CRT has been reported to be superior to radiation alone for local lesion control [5], potentially, it can improve survival time by causing metastatic tumour regression with systemic chemotherapy factored in CRT. However, drugs used in CRT need to undergo radiosensitization or radioenhancement and have lethal effect on cancer cells with minimum toxicity. Additionally, oral treatment of cancer is an attractive modality, it is easy to administer, to control complications and can be given in outpatient

*Corresponding author: Hiroaki Iwase, MD, Department of Gastroenterology, National Hospital Organization, Nagoya Medical Centre, 4-1-1 Sannomaru, Naka-ku, Nagoya, Aichi 460-0001, Japan. Tel.: +8152 9511111; Fax: +8152 9510664; Email: iwaseh@nnh.hosp.go.jp

Received 1 September 2016 Revised 25 November 2016 Accepted 7 December 2016 Published 21 December 2016

Citation: Iwase $H$, Shimada M, Hirashima N, Ryuge N, Urata N, Goto M, Kondo T, Tanaka D, Unita S, Kato E. Split-course chemoradiotherapy with S-1, a novel oral fluorouracil, and cisplatin for distant metastases of oesophageal cancer stage IVb. J Cancer Res Ther. 2017; 5(1):1-6. DOI: 10.14312/2052-4994.2017-1

Copyright: (c) 2017 Iwase H, et al. Published by NobleResearch Publishers. This is an open-access article distributed under the terms of the Creative Commons Attribution License, which permits unrestricted use, distribution and reproduction in any medium, provided the original author and source are credited. 
setting. Additionally, most patients prefer oral dosing instead of intravenous chemotherapy if efficacy is maintained. Previously, we assessed CRT alone or in combination with an oral fluoropyrimidine, uracil, and tegafur (UFT) for OC with distant metastasis [6, 7]. We found that CRT with UFT was more effective and no difference in severe complications compared to CRT without UFT [6]. A phase II study of CRT with cisplatin and UFT for distant metastatic OC showed an overall complete response (CR) rate of $8 \%$, but for primary lesions, the CR rate was $25 \%$, for lung metastases, the partial response (PR) rate was $18 \%$ and for liver metastases, the PR rate was $40 \%$. Complete resolution of dysphagia was achieved in $58 \%$ of patients. Most patients experienced severe dysphagia, but oral dosing was tolerated if administered as a powder with water [7]. S-1 is a fourth-generation, orally active fluoropyrimidine with reduced gastrointestinal toxicity [8]. Compared with 5-FU, the response rate (RR) in gastric cancer for S-1 was higher, while the incidence of toxicity was lower as compared with 5-FU [9]. Additionally, S-1 enhances radiotherapy and might prove to be an effective chemotherapeutic agent in a CRT setting [10].

Recently, we carried out a phase II study of CRT using S-1 and cisplatin for locally advanced OC [11]. The CRT regimen included two identical courses separated by a two-week interval to reduce toxicity and avoid prolonged hospitalization. Endoscopic CR rate for primary lesions were $91.7 \%$ for stage II, $71.9 \%$ for stage III, 60.6\% for stage Iva, and toxicity was tolerable. Disappearance of the primary lesion related to oesophageal obstruction can improve swallowing and quality of life. The regimen of split-course CRT with S-1 and cisplatin we have introduced for locally advanced $O C$ is anticipated to achieve better outcomes for patients with distant metastatic OC than hitherto reported [3-7]. In the present study, the outcomes of split-course CRT with S-1 and cisplatin in patients with distant metastatic OC are evaluated retrospectively with focus on palliation for dysphagia, complete response (CR) rate, toxicity, and survival time.

\section{Methods}

\section{Patients and disease locations}

During 2002 - 2014, 41 patients with distant OC metastasis and good Eastern Cooperative Oncology Group performance status (PS) of zero or one, but most with moderate to severe dysphagia, received split-course chemoradiotherapy with S-1 and cisplatin. Patients with distant OC and poor PS were given alternative treatment. All of the 41 patients, 37 male and 4 female listed in Table 1 were available for assessment. All patients had squamous cell carcinoma and most had moderate to severe dysphagia for solid foods or could eat only soft form of food. The degree of dysphagia was defined according to the modification of the method described by Coia, et al. [12]. Briefly, patient can eat solids without dysphagia (asymptomatic), eat solids with dysphagia, eat soft or pureed foods only, drink liquid only, no swallowing ability at all.

Thirty-eight patients had metastasis in the lymph nodes, and 16 of these had distant lymph node metastasis (M1b-Lym). The lungs $(n=15)$, liver $(n=7)$, bone $(n=$
Table 1 Patients' demography.

\begin{tabular}{|c|c|}
\hline \multicolumn{2}{|c|}{ Demography results } \\
\hline Age, median (range) year & $67(51-80)$ \\
\hline Gender, Male/Female & $37 / 4$ \\
\hline \multicolumn{2}{|l|}{ Performance status } \\
\hline 0 & $26(63.4 \%)$ \\
\hline 1 & $15(36.6 \%)$ \\
\hline \multicolumn{2}{|l|}{ Location } \\
\hline Upper & $7(17.1 \%)$ \\
\hline Middle & $24(58.5 \%)$ \\
\hline Lower & $10(24.4 \%)$ \\
\hline \multicolumn{2}{|l|}{ Organ of metastasis } \\
\hline Lung & $15(36.6 \%)$ \\
\hline Liver & $7(17.1 \%)$ \\
\hline Bone & $4(9.8 \%)$ \\
\hline Other & $4(9.8 \%)$ \\
\hline \multicolumn{2}{|l|}{ N stage (lymph node metastses) } \\
\hline No & $3(7.3 \%)$ \\
\hline N1 & $22(53.7 \%)$ \\
\hline M1 & $16(39.0 \%)$ \\
\hline \multicolumn{2}{|l|}{ T stage } \\
\hline $\mathrm{T} 2$ & $2(48.8 \%)$ \\
\hline T3 & $21(51.2 \%)$ \\
\hline $\mathrm{T} 4$ & $18(43.9 \%)$ \\
\hline \multicolumn{2}{|l|}{ Initial swallowing status } \\
\hline Asymptomatic & $3(7.3 \%)$ \\
\hline Eat solids with some dysphagia & $17(41.5 \%)$ \\
\hline Eat soft or purred food only & $16(39.0 \%)$ \\
\hline Drink liquids only & $5(12.2 \%)$ \\
\hline No swallowing at all & 0 \\
\hline
\end{tabular}

4) were the predominant organs of distant metastasis. At our institution, OC extent was determined by physical examination, barium swallow test, digestive endoscopy, or computed tomography (CT) of the neck, chest, and abdomen. Bone scan, endoscopic ultrasonography, and bronchoscopy were carried out when necessary. Similarly, tumour, node, metastasis (TNM) stage [13] before treatment was determined by $\mathrm{CT}$.

\section{Treatment schedule}

The treatment schedule is outlined in Figure 1. If a patient could not swallow the S-1 capsule, then S-1 was administered orally in powder form. CRT included two identical courses separated by a two-week interval. A treatment course comprised 30-Gy radiotherapy over three weeks (2 Gy per fraction, five times a week), with daily oral S-1 (70 mg/m²/day, maximum dose $100 \mathrm{mg} /$ day) for two weeks from Day 1, and a $24 \mathrm{~h}$ cisplatin infusion (70 $\mathrm{mg} / \mathrm{m}^{2}$, maximum dose $100 \mathrm{mg}$ ) on Day 8. Supraclavicular and cervical lymph nodes were generally included when the primary tumour was located above the carina; celiac lymph nodes were included for lesions distal to the 
primary tumour. The dose for the spinal cord was kept below 46 Gy. A reduced dose of S-1 (70\%) and cisplatin (70\%) were administered for patients over the age of 75 . A further $25 \%$ reduced dose adjustment was made for S-1 and cisplatin in the subsequent course if more than grade 3 toxicity occurred during the initial course. Patients could leave hospital during the two-week interval of CRT if they wished. For Patients who showed an objective response to the CRT, chemotherapy with S-1 and cisplatin was done followed by 21 days without drugs in a five-week cycle.

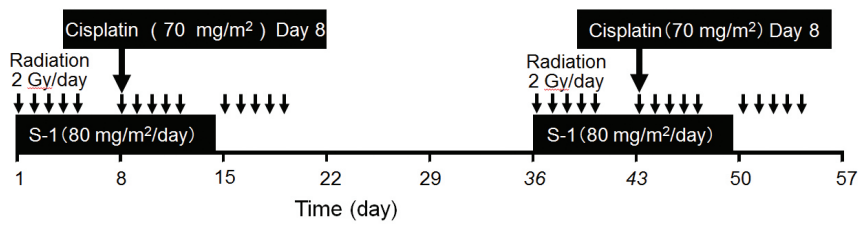

Figure 1 Treatment schedule for split-course concurrent chemoradiotherapy with S-1 and cisplatin.

\section{Evaluations}

Treatment toxicity was evaluated according to the National Cancer Institute's Common Toxicity Criteria [14]. Clinical response was assessed according to Response Evaluation Criteria in Solid Tumours (RECIST) $[15,16]$. Primary lesions were estimated by endoscopy according to the response evaluation criteria of the Japanese Society for Oesophageal Diseases [17]. Swallowing function, toxicity, response rates were determined by retrospective chart reviews. Similarly, the overall survival (OS) and progression free survival (PFS) rates were determined by retrospective chart review.

\section{Ethical considerations}

This retrospective evaluation was carried out with strict adherence to the Helsinki Declaration at all times. Likewise, our study protocol was reviewed and approved by the Institutional Review Board of the National Hospital Organization, Nagoya Medical Centre.

\section{Statistics}

The outcomes were evaluated in January 2016. The time intervals were calculated from the first day of treatment until the time of death or the last follow-up day. Dysphagiafree survival, OS, and PFS curves were plotted according to the Kaplan-Meier estimator graphs.

\section{Results}

\section{The overall treatment outcomes}

Among the 41 patients who completed the treatment course, $29(70.7 \%)$ received the planned treatment dose. Reductions in S-1 and/or cisplatin doses were necessary in $12(29.3 \%)$ patients during the subsequent course. The 34 of 41 patients who completed the CRT course received a total of 91 cycles of chemotherapy after CRT, median 2 cycles per patient, range, 1 to 8 cycles.

\section{Response rates}

The treatment efficacy or response rates are summarized in Table 2. Most patients responded better after the completion of CRT following chemotherapy. The overall response rates were as follows. Complete response (CR), 9 patients (22.0\%), partial response (PR) 18(43.9\%), and stable disease (SD) 10 (24.4\%). progressive disease (PD) occurred in 4 patients (9.8\%) at CRT completion. Endoscopic CR and response rates were $65.9 \%$ and $92.7 \%$, respectively. For lung metastasis, CR and response rates were $20.0 \%$ and $26.7 \%$, respectively; for liver metastasis, $14.3 \%$ and 28.6 $\%$, respectively. Metastases to other organs included one colon metastasis and one thyroid gland metastasis, which were eradicated by the treatment.

Table 2 Treatment efficacy in terms of response rates.

\begin{tabular}{lcccc}
\hline \multicolumn{1}{c}{ Location } & $\begin{array}{c}\text { Complete } \\
(\%)\end{array}$ & $\begin{array}{c}\text { Partial } \\
(\%)\end{array}$ & $\begin{array}{c}\text { Stable } \\
\text { disease } \\
(\%)\end{array}$ & $\begin{array}{c}\text { Progressive } \\
\text { disease (\%) }\end{array}$ \\
\hline Primary lesion $(\mathrm{n}=41)$ & $27(65.9)$ & $11(26.8)$ & $2(4.9)$ & $1(2.4)$ \\
Lung $(\mathrm{n}=15)$ & $3(20.0)$ & $4(26.7)$ & $6(40.0)$ & $2(13.3)$ \\
Liver $(\mathrm{n}=7)$ & $1(14.3)$ & $2(28.6)$ & $3(42.9)$ & $1(14.3)$ \\
Bone $(\mathrm{n}=4)$ & $0(0.0)$ & $0(0.0)$ & $3(7.3)$ & $1(2.4)$ \\
Others $(\mathrm{n}=4)$ & $2(50.0)$ & $1(25.0)$ & $1(25.0)$ & $0(0.0)$ \\
M1 lymph node $(\mathrm{n}=16)$ & $3(18.8)$ & $4(25.0)$ & $5(31.2)$ & $4(25.0)$ \\
\hline
\end{tabular}

Improvement of swallowing function

Swallowing function status of all patients at the initial and the final CRT completion are shown in Table 3. Twentyone patients (51.2\%) were asymptomatic at the initial CRT completion, 31 (75.6\%) became asymptomatic by the final CRT completion following chemotherapy. Asymptomatic patients regained normal swallowing function and could eat solid food with some dysphagia. The duration time curve for normal swallowing function is shown in Figure2.

Table 3 Swallowing conditions at the initial and at the completion of the final chemoradiotherapy (CRT).

\begin{tabular}{lcc}
\hline Swallowing status & $\begin{array}{c}\text { At completion of } \\
\text { the first CRT }-n \\
(\%)\end{array}$ & $\begin{array}{c}\text { At completion of } \\
\text { the final CRT }-n \\
(\%)\end{array}$ \\
\hline Asymptomatic & $21(51.2)$ & $31(75.6)$ \\
Eat solids with some dysphagia & $15(36.6)$ & $7(17.1)$ \\
Eat soft or purred food only & $3(7.3)$ & $1(2.4)$ \\
Drink liquids only & $1(2.4)$ & $1(2.4)$ \\
No swallowing ability & $1(2.4)$ & $1(2.4)$ \\
\hline
\end{tabular}

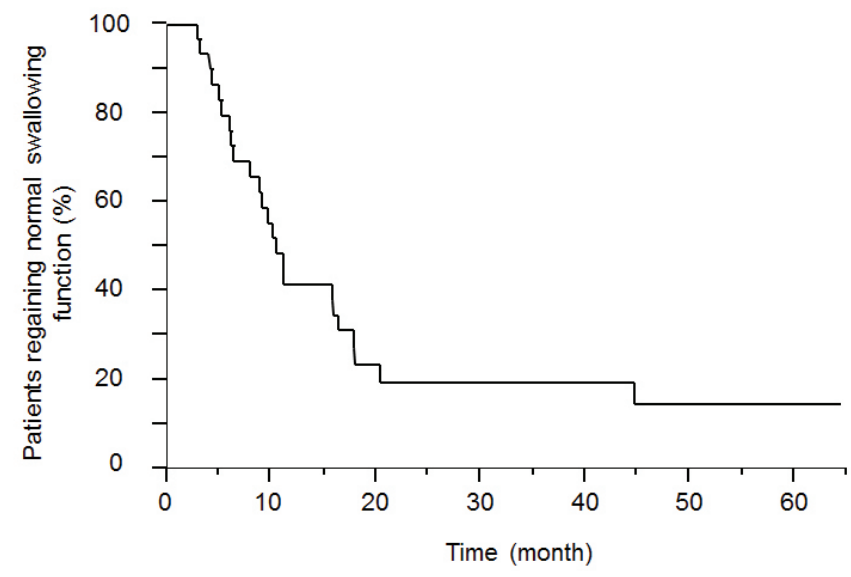

Figure 2 The time course of regaining normal swallowing function in patients with metastatic oesophagus cancer. 
The median time to regain normal swallowing function was 10.1 months. The improvement in swallowing lasted without parenteral nutritional support until near death in 25 patients $(61.0 \%)$.

\section{Survival rates and PFS values}

The OS and PFS curves for all patients are presented in Figure 3. MST was 12 months and the median PFS was 5.2 months. Four patients who achieved CR were still alive at the time of this writing, more than 3 years after regaining normal swallowing function.

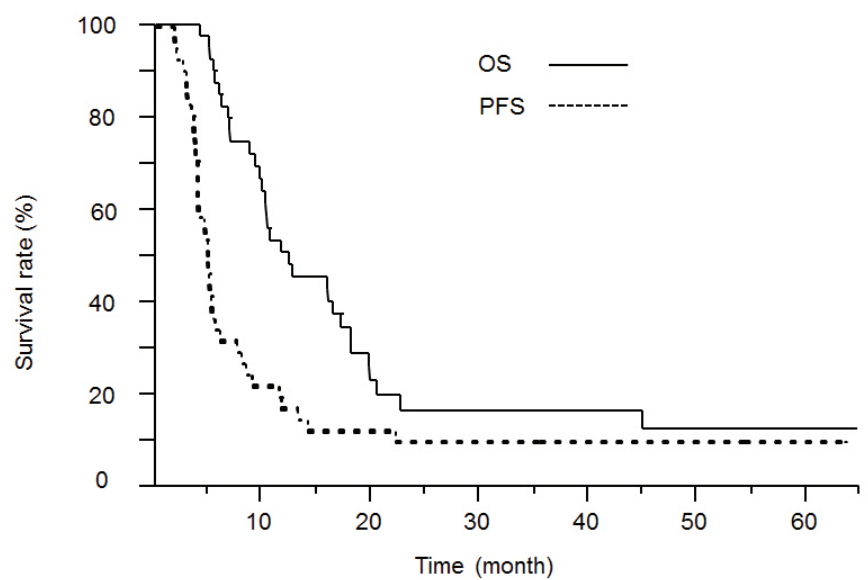

Figure 3 The overall survival (OS) and progression free survival (PFS) plots for all 41 patients.

\section{Adverse events}

Toxicity observations are summarized in Table 4. In the category of haematological toxicity the most frequent AEs were grade 3 and 4 neutropenia (neutrophils) (29.2\%),

Table 4 Treatment toxicity by grade.

\begin{tabular}{|c|c|c|c|c|c|}
\hline & $G 2(n)$ & G3 (n) & G4 (n) & G2 (\%) & G3/G4 (\%) \\
\hline \multicolumn{6}{|l|}{ Haematological toxicity } \\
\hline Neutrophils & 25 & 9 & 3 & 60.9 & 29.2 \\
\hline Platelets & 13 & 3 & 1 & 31.7 & 9.8 \\
\hline Haemoglobin & 10 & 2 & 1 & 24.3 & 7.3 \\
\hline \multicolumn{6}{|l|}{ Non-haematological toxicity } \\
\hline Nausea & 13 & 2 & 0 & 31.7 & 4.9 \\
\hline Vomiting & 6 & 1 & 0 & 14.6 & 2.4 \\
\hline Annorexia & 5 & 1 & 0 & 12.2 & 2.4 \\
\hline $\begin{array}{l}\text { Oesophageal } \\
\text { discomfort }\end{array}$ & 5 & 0 & 0 & 12.2 & 0 \\
\hline Oral mucositis & 4 & 1 & 0 & 9.8 & 2.4 \\
\hline Fatigue & 4 & 2 & 0 & 9.8 & 4.9 \\
\hline Creatinine & 3 & 1 & 0 & 7.3 & 2.4 \\
\hline Diarrhoea & 2 & 0 & 0 & 4.9 & 0 \\
\hline Constipation & 2 & 0 & 0 & 4.9 & 0 \\
\hline Hyperpigmentation & 1 & 0 & 0 & 2.4 & 0 \\
\hline Sensory neutropathy & 1 & 0 & 0 & 2.4 & 0 \\
\hline Sensory neutropathy & 1 & 0 & 0 & 2.4 & 0 \\
\hline
\end{tabular}

thrombocytopenia (platelets) (9.8\%), and anaemia (haemoglobin) (7.3\%). Non-haematological AEs were generally mild, and included grade 2 nausea (31.7\%), oesophageal discomfort (12.2\%), oral mucositis (9.8\%), and renal dysfunction (7.3\%). AEs in the initial course of CRT remitted during the two-week interval.

\section{Discussion}

Hitherto, for distant metastatic OC, combination chemotherapy has been a widely applied option. In fact, most combination chemotherapy regimens achieve moderate response rates, some cause severe toxicity, and even the most intensive regimens have been unsuccessful in producing a CR or leading to a normal swallowing function in the treated patients. The MST has not been longer than seven months $[18,19]$. However, treatment decisions for patients with distant metastatic OC should take into account both the improvement in survival and impact on patient's quality of life, especially dysphagia, which most patients with advanced OC have. CRT appears to be a promising treatment option for OC, which is often difficult to treat [20]. The combination of 5-FU and cisplatin with radiotherapy for the treatment of locally advanced OC has been used worldwide. Conventional CRT with 5-FU and cisplatin has been associated with moderate to severe side effects [21]. Excessive toxic side effects significantly limit patient's compliance, who are often weak and malnourished.

The cytotoxic effect of 5-FU increases when drug exposure is prolonged beyond the cell-cycle time. Therefore, the duration of administration was an important factor in determining the amount of synergistic effect between 5-FU and irradiation. However, continuous intravenous infusion of 5-FU can be replaced by long-term daily oral administration of S-1. As S-1 is administered orally, it is convenient for divided radiotherapy [10]. Further, a significant safety advantage was observed in the S-1 plus cisplatin regimen as compared to the 5-FU plus cisplatin regimen in advanced gastric or gastroesophageal adenocarcinoma studies [22]. Furthermore, S-1 plus cisplatin regimen provides a safe doublet platform to add one or more other drugs [23]. The daily dose of S-1 did not produce severe side effects when administered in combination with cisplatin infusion or with radiotherapy [11]. The slow infusion of cisplatin over a $24 \mathrm{~h}$ instead of bolus infusion was a relevant approach to minimize gastrointestinal side effects like nausea, diarrhoea, renal dysfunction and loss of appetite [7, 11, 23]. Further, a 3 week course of CRT was tolerated by even patients who had a poor prognosis.

We have introduced a split-course scheme of CRT, separated by a two-week interval to shorten a long ( $>4$ weeks) hospital stay. The most common AE was neutropenia, which was not fatal. The non-haematological side effects, which is important in maintaining CRT, were mild and well tolerated. Adherence to CRT results in enhanced treatment outcome, it is a gentle regimen for patients who are weak or elderly, and ensures good compliance. The overwhelming majority of the patients were well after the first course of CRT. Following a two-week rest, they received one more courses to provide better efficacy outcomes. The theoreti- 
cal advantage of split-course radiation on the tumour itself may not show the desired effect unless clinically relevant doses of radiation over 56 Gy can be applied safely. Wobbes et al. [24] reported that split-course radiation in combination with cisplatin was very well tolerated. Clinically relevant radical doses of split-course radiation with S-1 plus cisplatin lead to a higher rate of CR on primary lesions and regaining of swallowing function in most patients with dysphagia. Swallowing function improved at the completion of the final CRT course and was sustained until near death in most patients. Long-term normal swallowing function always requires local control of cancer, which CRT followed by S-1 and cisplatin appeared to be very effective. Indeed, to improve the survival of patients with distant metastatic $\mathrm{OC}$, it is essential to eradicate all of the metastatic lesions.

Previously [25], we reported a CR outcome in an OC case with metastasis to the colon. The outcome in that case encouraged us to extend the strategy to other patients with metastatic OC. In this study, except for bone marrow metastasis, we achieved moderate response rates for the metastases and the overall median survival time was 12 months. For patients who showed an objective response at the CRT, it is important to continue the systemic chemotherapy together with CRT to decrease distant metastases and improve survival time.

In spite of high dose radiation together with chemotherapy like bolus mitomycin $\mathrm{C}$ and continuous infusion of 5-FU are reported to provide a rapid and an initial improvement of dysphagia in nearly $90 \%$ of patients with OC [12], but radical doses of radiation treatment over five to six weeks might be inappropriate for patients with distant metastatic OC who are expected to have a short survival times. Further, there is increasing evidence that low dose radiotherapy together with chemotherapy provides good palliation of dysphagia with limited side effects $[26,27]$. Harvey et al. [26] described applying CRT with median radiation dose of 35 Gy in 15 fractions and a concurrent single course of 5-FU based chemotherapy. The treatment was well tolerated, with only $5 \%$ of patients failing to complete the treatment course. The treatment mortality rate was 6\%, MST was seven months. Seventy-eight patients had improved swallowing, and 41\% regained normal swallowing. Likewise, Hayter [27] reported that radiotherapy with $30-$ Gy in 10 fractions with synchronous 5-FU and mitomycin C given in the first week of treatment achieved a CR rate of $68 \%$ with limited adverse side effects. Palliative CRT can lead to normal swallowing function in many patients with very modest side effects, but there has been no report on improving the survival time. At present, newer, well-designed, tolerable CRT regimens using more radioenhancement and cytotoxic drugs are expected for metastatic OC.

Recently, clinical studies on CRT with S-1 and cisplatin have been undertaken to treat certain forms of advanced cancers. Kaira et al. [28] have described CRT with S-1 plus cisplatin for locally advanced squamous cell cancer and presented an impressive efficacy outcome of $87.8 \%$ and a MST of 29.7 months with limited side effects. Likewise, efficacy outcomes for CRT with S-1 plus cisplatin for gastric, head and neck cancers are expected [29,30], and are considered as candidates for palliative therapy. This treatment strategy might be recommended for any other advanced solid cancer.

\section{Conclusion}

In patients with metastatic OC, our split-course CRT involved radical doses of radiation together with oral anticancer drugs S-1 and a $24 \mathrm{~h}$ cisplatin infusion. The treatment was well tolerated and led to normal or near-normal swallowing function in the majority of the patients, with very limited side effects. Potentially, this strategy should become a standard treatment option, an alternative to conventional palliative therapy for patients with distant metastatic OC. However, one major limitation of this investigation might be that it looks more like a survey than a clinical trial report. We believe that future trials in large cohorts of patients are needed to support the outcomes of this study.

\section{Conflict of interest}

No external funding was received for this study. Further, the authors declare having no conflict of interest in connection with the publication of this manuscript.

\section{References}

[1] Daly JM, Karnell LH, Menck HR. National Cancer Data Base report on esophageal carcinoma. Cancer. 1996; 78(8):1820-1828.

[2] Mariette C, Piessen G, Triboulet JP. Therapeutic strategies in oesophageal carcinoma: Role of surgery and other modalities. Lancet Oncol. 2007; 8(6):545-553.

[3] Besharat S, Jabbari Ali, Semnani S, Keshtkar A, Marjani J. Inoperable esophageal cancer and outcome of palliative care. World J Gastroenterol. 2008; 14(23):3725-3728.

[4] O'Rourkke IC, Tiverk K, Bull C, Gebski V, Langlands A. Swallowing performance after radiation therapy for carcinoma of the esophagus. Cancer. 1988; 61(10):2002-2006.

[5] Herskovic A, Martz K, al-Sarraf M, Leichman L, Brindle J, et al. Combined chemotherapy and radiotherapy compared with radiotherapy alone in patients with cancer of the esophagus. N Engl J Med. 1992; 326(24):1593-1598.

[6] Iwase $\mathrm{H}$, Morise $\mathrm{K}$. Chemotherapy combined with radiation for advanced esophageal carcinoma. Intern Med. 1992; 31:1433-1434.

[7] Iwase H, Shimada M, Nakamura M, Nakarai K, lyo T, et al. Concurrent chemoradiotherapy for locally advanced and metastatic esophageal cancer: Long-term results of a phase II study of UFT/CDDP with radiotherapy. Int J Clin Oncol. 2003; 8(3):305-311.

[8] Shirasaka T, Shimamoto $Y$, Ohshimo H, Yamaguchi M, Kato T, et al. Development of a novel form of an oral 5-fluorouracil derivative (S-1) directed to the potentiation of the tumor selective cytotoxicity of 5 -fluorouracil by two biochemical modulators. Anticancer Drugs. 1996; 7(5):548-557.

[9] Boku N, Yamamoto S, Fukuda H, Shirao K, Doi T, et al. Fluorouracil versus combination of irinotecan plus cisplatin versus $\mathrm{S}-1$ in metastatic gastric cancer: A randomised phase 3 study. Lancet Oncol. 2009; 10(11):1063-1069.

[10] Harada K, Kawaguchi S, Supriatno, Kawashima Y, Yoshida H, et al. S-1, an oral fluoropyrimidine anti-cancer agent, enhanced radiosensitivity in a human oral cancer cell line in vivo and in vitro: involvement possibility of inhibition of survival signal, Akt/PKB. Cancer Lett. 2005; 226(2):161-168.

[11] Iwase H, Shimada M, Tsuzuki T, Hirashima N, Okeya M, et al. Concurrent chemoradiotherapy with a novel fluoropyrimidine, S-1, and cisplatin for locally advanced esophageal cancer: Long-term results of a phase II trial. Oncology 2013; 84(6):342-349.

[12] Coia LR, Soffen EM, Schultheiss TE, Martin EE, Hanks GE. Swallowing function in patients with esophageal cancer treated with concurrent radiation and chemotherapy. Cancer. 1993; 71(2):281-286.

[13] Sobin LH, Witteckind C. International Union against Cancer (UICC) TNM classification of malignant tumors, 5th edn. WileyLiss. 1997. 
[14] Trotti A, Byhardt R, Stetz J, Gwede C, Corn B, et al. Common toxicity criteria: Version 2.0. an improved reference for grading the acute effects of cancer treatment: Impact on radiotherapy. Int J Radiat Oncol Biol Phys. 2000; 47(1):13-47.

[15] Therasse P, Arbuck SG, Eisenhauer EA, Wanders J, Kaplan RS, et al. New guidelines to evaluate the response to treatment in solid tumors. European Organization for Research and Treatment of Cancer, National Cancer Institute of the United States, National Cancer Institute of Canada. J Natl Cancer Inst. 2000; 92(3):205-216.

[16] Nishino M, Jagannathan JP, Ramaiya NH, Van den Abbeele AD. Revised RECIST guideline version 1.1: What oncologists want to know and what radiologists need to know. AJR Am J Roentgenol. 2010; 195(2):281289.

[17] Japanese Society for Esophageal Diseases: Guidelines for Clinical and Pathologic Studies on Carcinoma of the Esophagus, Ninth Edition. Kanehara \& Co., Ltd., Tokyo. 2001.

[18] Grünberger B, Raderer M, Schmidinbrt M, Hejina M. Palliative chemotherapy for recurrent and metastatic esophageal cancer. Anticancer Research. 2007; 27(4C):2705-2714.

[19] Al-Batran SE, Ajani JA. Impact of chemotherapy on quality of life in patients with metastatic esophagogastric cancer. Cancer. 2010; 116(11):2511-2518.

[20] Tanaka T, Fujita H, Matono S, Nagano T, Nishimura K, et al. Outcomes of multimodality therapy for stage IVb Esophageal Cancer with distant organ metastasis (M1-Org). Dis Esophagus. 2010; 23(8):646-651.

[21] Ohtsu A, Boku N, Muro K, Chin K, Muto M, et al. Definitive chemoradiotherapy for T4 and/or M1 lymph node squamous cell carcinoma of the esophagus. J Clin Oncol. 1999; 17(9):2915-2921.

[22] Ajani JA, Rodriguez W, Bodoky G, Moiseyenko V, Lichinister M, et al. Multicenter phase III comparison of cisplatin/S-1 with cisplatin/infusion fluorouracil in advanced gastric or gastroesophageal adenocarcinoma study: The FLAGS trial. J Clin Oncol. 2010; 28(9):1547-1553.

[23] Iwase H, Shimada M, Tsuzuki T, Ina K, Sugihara M, et al. A phase II multi-center study of triple therapy with paclitaxel, S-1 and cisplatin in patients with advanced gastric cancer. Oncology. 2011; 80(1-2):76-83.

[24] Wobbes Th, Baron B, Paillot B, Jacob JH, Haegele $P$, et al. Prospective randomised study of split-course radiotherapy versus cisplatin plus split-course radiotherapy in inoperable squamous cell carcinoma of the oesophagus. Eur J Cancer. 2001; 37(4):470-477.

[25] Iwase H, Indo T, Shimada M, Tsuzuki T, Nakarai K, etal. Esophageal cancer with colonic metastasis successfully treated by chemoradiotherapy followed by chemotherapy with S-1 and cisplatin. Int J Clin Oncol 2004; 9(5):398-402.

[26] HarveyJA, BessellJR, Beller E, Thomas J, Gotley DC, et al. Chemoradiation therapy is effect for the palliative treatment of malignant dysphagia. Dis Esophagus. 2004: 17(3):260-265.

[27] Hayter CR, Huff-Winters C, Paszat L, Youssef YM, Shelley WE, et al. A prospective trial of short-course radiotherapy plus chemotherapy for palliation of dysphagia from advanced esophageal cancer. Radiother Oncol. 2000; 56(3):329-333.

[28] Kaira K, Tomizawa $Y$, Yoshino $R$, Matsuura M, Iwasaki $Y$, et al. Phase II study of oral S-1, and cisplatin with concurrent radiotherapy for locally advanced non-small-cell lung cancer. Lung Cancer. 2013; 82(3):449454.

[29] Takahashi T, Saikawa Y, Takaishi H, Takeuchi H, Wada N, et al. Phase I study of neoadjuvant chemoradiotherapy consisting of S-1 and cisplatin for patients with resectable advanced gastric cancer (KOGC01). Anticancer Res. 2011; 31(9):3079-3084.

[30] Tahara M, Minami H, Kawashima M, Kawada K, Mukai H, et al. Phase I trial of chemoradiotherapy with the combination of S-1 plus cisplatin for patients with unresectable locally advanced squamous cell carcinoma of the head and neck. Cancer Sci. 2011; 102(2):419-424. 